\title{
Differences of enamel print patterns between buccal and lingual surfaces of extracted permanent maxillary first premolar
}

\author{
Sheng Cheng Soo ${ }^{1}$, Nani Murniati², Andriani Harsanti ${ }^{3}$, Yuti Malinda ${ }^{2}$, Fahmi \\ Oscandar ${ }^{1 *}$ \\ ${ }^{1}$ Department of Dentomaxillofacial Radiology, Faculty of Dentistry Universitas Padjadjaran, \\ Indonesia \\ ${ }^{2}$ Department of Oral Biology, Faculty of Dentistry Universitas Padjadjaran, Indonesia \\ ${ }^{3}$ Department of Orthodontics, Faculty of Dentistry Universitas Padjadjaran, Indonesia
}

\begin{abstract}
Introduction: Ameloglyphics is the study of enamel print patterns on the tooth surface. Enamel print patterns are unique to each individual as they differ on each tooth. The similarity of tooth prints on buccal and lingual surfaces remains questionable as no research has been conducted thus far. This research was aimed to investigate the differences between enamel print patterns on buccal and lingual surfaces of maxillary right first premolar. Methods: An analytic study was conducted by observing and analysing a total of 34 teeth samples using the purposive sampling method. The middle third of the buccal and lingual tooth surfaces was captured directly after magnified with a stereomicroscope. Photomicrographs of samples were then analysed using SourceAFIS biometric software. This software will process the image and shows patterns of enamel print. Afterwards, the enamel print patterns were observed and classified according to Manjunath Classification. Results: The results suggested that all 34 samples show a similarity of enamel print patterns on both the buccal and lingual surfaces. The interpretations of photomicrographs were statistically analysed using the Statistical Package of Social Science (SPSS) software. The similarity may be due to the same placement of developing tooth bud, temperature, environmental factor, genetics, pressure and nutrition to the ameloblasts cells in a tooth. Conclusion: Both buccal and lingual in the samples show high similarity due to the very high frequencies of similar sub-patterns occur on both surfaces. Tooth print can be used as a valuable tool in forensic science for personal identification with good prospects in the field of forensic dentistry.
\end{abstract}

Keywords: Enamel print pattern, permanent maxillary right first premolar, ameloglyphics.

p-ISSN: 1979-0201; e-ISSN: 2549-6212; Available from: http://jurnal.unpad.ac.id/pjd/article/view/21639

DOI: 10.24198/pjd.vol32no2.21639

Submission: May 25, 2019; Accepted: Jul 30, 2020; Published online: Jul 31, 2020

"Corresponding author: Fahmi Oscandar, Department of Dentomaxillofacial Radiology, Faculty of Dentistry Universitas Padjadjaran, Indonesia. Department of Dentomaxillofacial Radiology, Faculty of Dentistry Universitas Padjadjaran, Jalan Sekeloa Selatan I, Bandung, West Java, Indonesia, 40132. Phone: +6281214343669; Email: fahmi.oscandar@fkg.unpad.ac.id 


\section{INTRODUCTION}

Bodieswhich arewhollyor partiallyskeletonised due to numerous reasons have remained undiscovered either intentionally or unintentionally. Some bodies are found in the home of the deceased after months or even years, This discovery usually attracts media coverage, causing an uproar as well as indict sorrow on society. Mass disasters such as earthquakes, tsunamis or catastrophes like motor vehicles accidents are the main reason for the difficulty that might be found in body identification. ${ }^{1}$ Remains that are skeletonised or partially skeletonised require coronal investigation to answer umpteen social and legal questions. However, the autopsy is further complicated due to the effects of decomposition; consequently rendering some routine procedures, for instance, organ histology, less useful. ${ }^{2}$

Hence, forensic odontology, a field of dentistry that used to identify human, which involving application of dental sciences to the law, has been an integral part of the American judicial system for over three decades. ${ }^{3}$ Bansode et $\mathrm{al}^{4}$ stated that the standard identification uses fingerprints. However, this form of identification is not possible if there are no antemortem records. In cases where fingerprints are no longer obtainable, such as in the cases of burnt, decomposed or skeletonised remains, dental evidence plays a significant role in identifying human identity. ${ }^{5}$ As technologies progressed, dental evidence progressed as well, from using antemortem dental records such as denture and tooth fillings, bite marks, rugoscopy, cheiloscopy to enamel print to distinct individual. ${ }^{6}$

Enamel is the hardest tissue in the human body and manages to remain intact under extreme temperatures and time-invariance due to its content of mineral salts and their crystalline arrangement. Enamel develops from ectoderm and is a product of specialised epithelial cells known as ameloblast. During amelogenesis, the undulating course of ameloblast leads to the formation of a pattern by a series of adjacent enamel rod ends. ${ }^{7}$ Tooth prints are the term used to describe the enamel rod end patterns, while ameloglyphics is the term used for the study of patterns of enamel rods. Amelo means enamel and glyphic means carving. ${ }^{8}$
Besides, this technique is also simple, inexpensive, rapid and practical as nonprofessionals can also perform it. This method is usually recommended for individuals such as firefighters, soldiers, jet pilots, divers, and people who live or travel to politically unstable areas because they have higher risk involving in an accident due to their risky occupations. ${ }^{3}$

According to studies, tooth prints appear to be unique to an individual, with dissimilarities between those of different individuals and also the same individuals ${ }^{1}$, which composed of a combination of eight different subpatterns. ${ }^{9}$ However, the author was curious whether tooth prints will appear to be similar or not on different surfaces of the same tooth. Hence, the author would like to carry out an experiment to observe the differences of enamel print pattern on the buccal and lingual surfaces on the same tooth by using extracted permanent maxillary right first premolar. This research was aimed to investigate the differences between enamel print patterns on buccal and lingual surfaces of maxillary right first premolar. If both buccal and lingual surfaces do not have significant differences, the enamel print pattern can be used in identification purpose.

\section{METHODS}

The type of study was an analytical study which observes and analyses the stereo microscopical differences between enamel print patterns on buccal and lingual surfaces of extracted permanent maxillary right first premolar. The research was held in the Faculty of Dentistry Universitas Padjadjaran from October-December 2018.

The population and samples of this research were 34 post-extracted permanent maxillary right first premolars of individuals who came to Exodontics Clinic of Universitas Padjadjaran Dental Hospital from October 2018 to November 2018 with the inquiry for tooth extraction treatments (ethical clearance No. 1156/UN6.KEP/EC/2018). Teeth with caries, erosion, abrasion, restoration and fracture on the middle third of buccal and lingual surfaces were excluded from the study because of the alteration of surface enamel characteristics in such teeth. The genders of the samples were unknown. Sample taking technique used in this research was purposive sampling. 
Research procedure divided into preliminary and working stages. In the preliminary stage, the samples were collected based on inclusive and exclusive criteria. The inclusive criteria included were the middle third of buccal and lingual surfaces teeth must free of caries, erosion, abrasion, restoration and fracture. The exclusion criteria were gender, races and age of sample population. These samples were cleaned with distilled water and dispensed in $10 \%$ formalin. Later, the samples were open-air dried, scaled and labelled accordingly.

In the working stage, the crown was divided with a ruler into three regions, partly incisal third, middle third and cervical third and marked (Figure 1) with a pencil. Each dimension was equivalent to each other and was determined by using compasses. The middle third of the buccal and lingual surfaces of a tooth was selected for photomicrograph (Figure 2.A) as the enamel rods were least likely to show directional curves and entangling in the rod pattern.

Afterwards, the sample was placed on Nikon SMZ800 zoom stereomicroscope (Nikon Corporation, Tokyo, Japan) using removable adhesive tape. Nikon digital SLR camera D5100 (Nikon Corporation, Tokyo, Japan) is mounted and connected to the stereomicroscope. Photomicrograph of the middle section of samples were observed, captured directly after zoom in with stereomicroscope under magnification of X400 (Figure 2.B) and transferred to a laptop in the format of JPEG (Joint Photographic Experts Groups). The photomicrograph of enamel print was analysed by using SourceAFIS biometric-based software written by Robert Važan to identify enamel rod end patterns (Figure 2.C). The processed image or the patterns of enamel print are classified according to Manjunath Classification', as shown on Figure 3. Only one observer was involving in classification of the enamel print pattern due to limited observer equipped with knowledge on enamel print patterns.

Tooth prints obtained from 34 samples (total of 68 tooth prints) were analysed for similarities among buccal and lingual surfaces of a tooth for these samples. The interpretations of photomicrographs were statistically analysed by using Statistical Package for Social Science (SPSS) version 23.0 (SPSS Inc., Chicago, United States) for data analysis.

\section{RESULTS}

Photomicrographs of tooth print on the buccal surface and lingual surface of the same tooth are shown in Figure 4. Both surfaces were made up of wavy branched and whorl open with a different arrangement. This result showed that

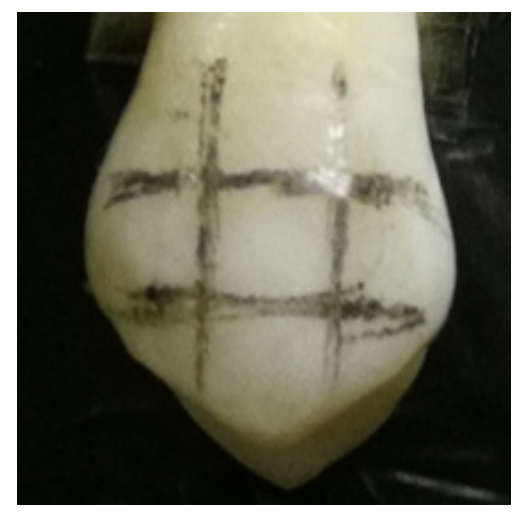

Figure 1. The crown divided into 3 regions partly incisal third, middle third, and cervical third, then marked

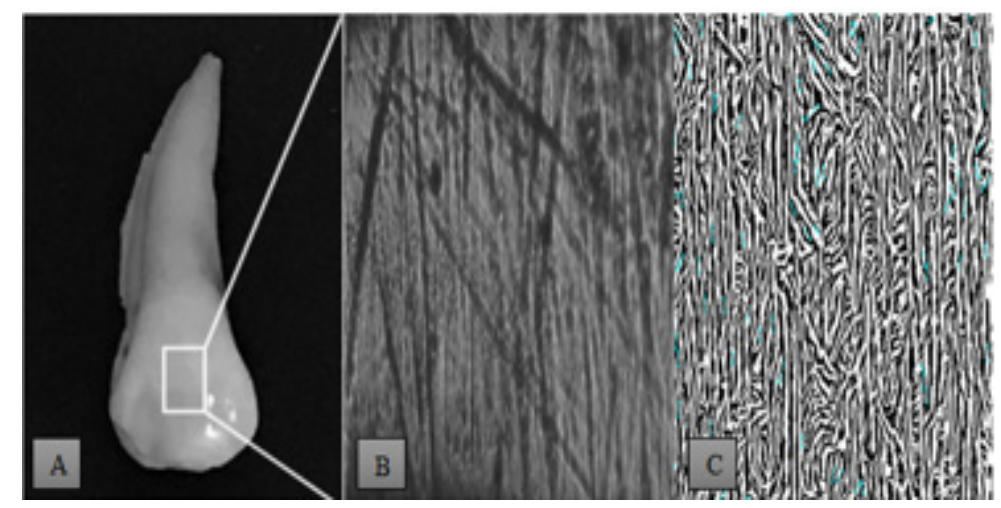

Figure 2. Result of enamel print pattern on tooth surface; A. Photo of maxillary first premolar; B. Image of middle third crown after being magnified with stereomicroscope (magnification of 400x); C. Enamel print pattern after being analysed with SourceAFIS biometric-based software 


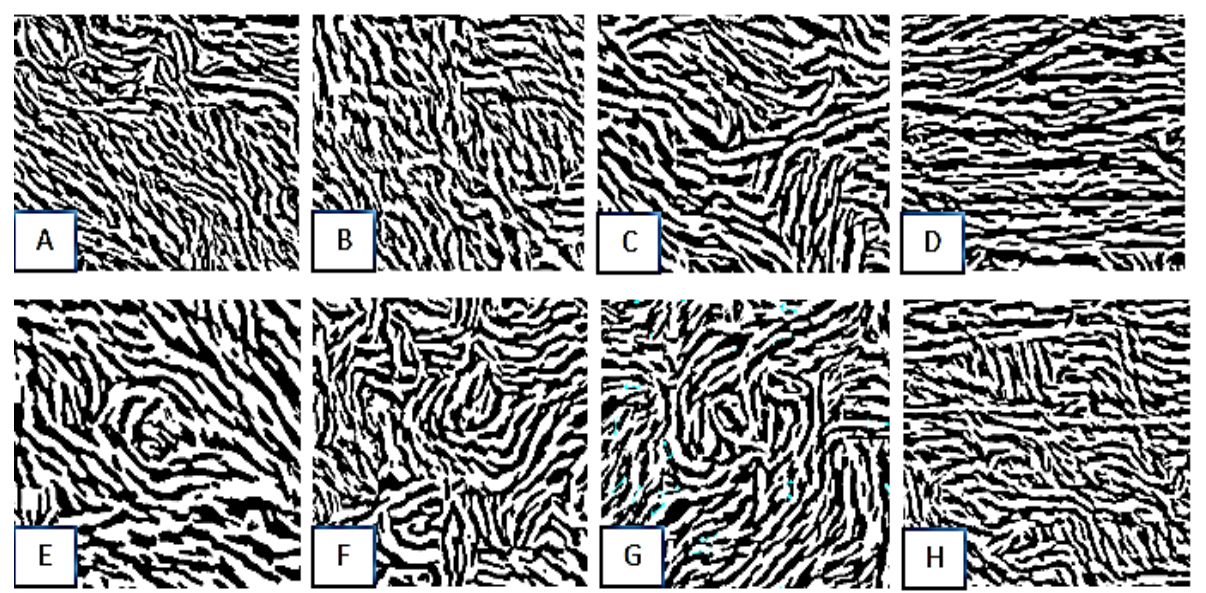

Figure 3 Manjunath classification ${ }^{10}$ of enamel prints; A. wavy-branched; B. wavy-unbranched; C. linear-branched; D. linearunbranched; E. whorl-open; F. whorl-closed; G. loop and; H. stem-like

even though the enamel print patterns of buccal and lingual surfaces were located in the same sample, however, they may not have the same arrangement, with the occurence or absence frequency of the similar sub-patterns in both surfaces were found to be very high.

Figure 5 shows the total number of subpattern on buccal and lingual surfaces among these eight types of sub-patterns. There were a total of 145 (n-value) sub-patterns combinations found from the samples collected. Each sample has a combination of a few sub-patterns, with a minimum of at least two sub-patterns. For example, more than one sub-pattern can be found on enamel print patterns on the buccal of a sample. The different patterns of enamel print were observed with varying degrees of dominance. The most dominant pattern of enamel prints was the wavy-branched; in decreasing order of dominance was the whorl-open, linear-branched. The less common sub-pattern was linear-unbranched and whorl-closed which have the same number of subpattern, followed by loop, wavy-unbranched and stem-like.
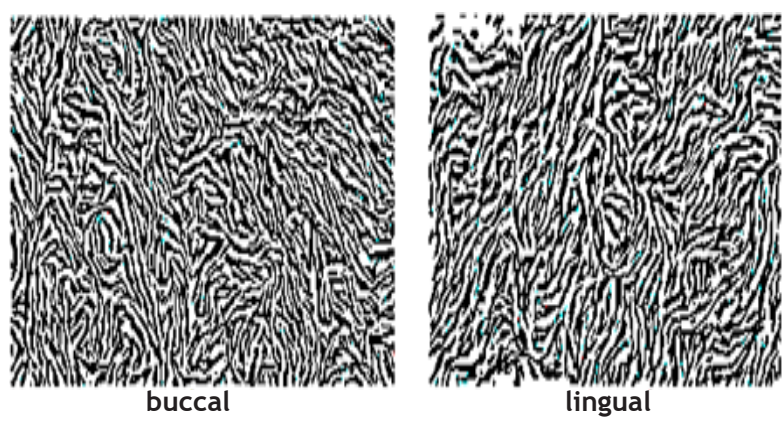

Figure 4. Photomicrographs of tooth print on buccal (left) and lingual (right) surfaces of a same tooth of sample number (\#10) under the magnification of $400 x$

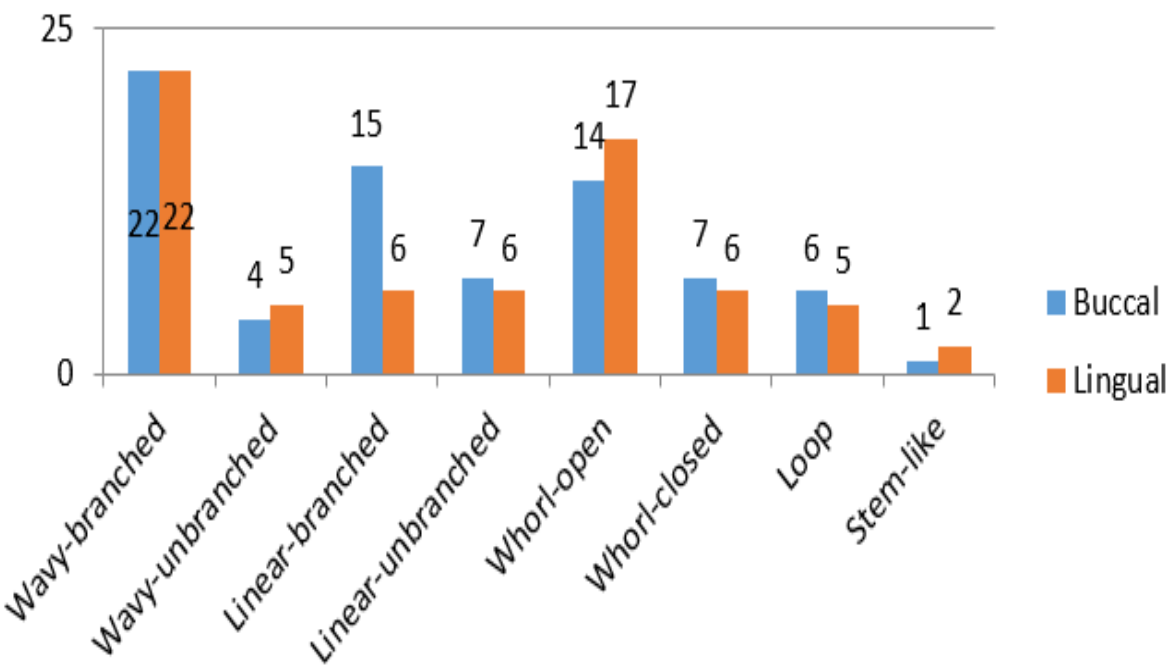

Figure 5. Frequency distribution of enamel print patterns on buccal and lingual surfaces based on Manjunath classification 
Table 1 and 2 shows the normality and Levene's test results of the numerical variables normality. In the Shapiro-Wilk test, if the value of Sig. $\geq 0.05$, then the data is normally distributed. $^{2}$ Based on the Shapiro-Walk test results, the significant value was less than 0.05 for each sample; thus, the data was not normally distributed.

The Levene's test suggested that if the $\mathrm{p}$-value $\geq$ alpha (0.05), then the variance of the two data is homogeneous. Based on the Levene's test results, the variance of group scores data was homogeneous because of the $p$-value $\geq$ alpha (0.05).

Table 1. Normality test of 34 samples

\begin{tabular}{llllll}
\hline \multicolumn{2}{c}{ Group } & \multicolumn{5}{c}{ Shapiro-Wilk } \\
\hline Sample & Statistic & df & Sig. & $\begin{array}{l}\text { Alpha } \\
\text { (a) }\end{array}$ & Conclusion \\
\hline Buccal & 0.418 & 8 & 0.000 & 0.05 & Not Normal \\
Lingual & 0.566 & 8 & 0.000 & & Not Normal \\
\hline
\end{tabular}

Table 2. Levene test of 34 samples

\begin{tabular}{lllcl}
\hline & Levene Statistic & Sig. & $\begin{array}{c}\text { Alpha } \\
\text { (a) }\end{array}$ & Conclusion \\
\hline Sample & 1.577 & 0.230 & 0.05 & Homogeneous \\
\hline
\end{tabular}

Table 3. Mann-Whitney test of 34 samples

\begin{tabular}{ll}
\hline & Sample \\
\hline Mann-Whitney U & 28.000 \\
Wilcoxon W & 64.000 \\
Z & -.620 \\
Asymp. Sig. (2-tailed) & .535 \\
Exact Sig. [2*(1-tailed Sig.)] & 0.721 \\
\hline
\end{tabular}

Independent t-test is performed if the data is normally distributed while Mann Whitney non-parametric test is performed if the data is not normally distributed. ${ }^{11}$ We evaluate whether the means for two independent groups were significantly different from each other. The independent-samples t-test is commonly referred to as a between-groups design, and can also be used to analyse control and experimental group. With an independent-samples t-test, each case must have scores on two variables, the grouping. Based on the results of the normality and homogeneity tests can be seen that there was a violation of the independent parametric t-test assumption; therefore, the test was carried out using the Mann Whitney non-parametric test. ${ }^{12}$
Based on the Mann-Whitney test with $a=$ 0.05 , the $p$-value in the whole sample was $\geq 0.05$; thus, it was not significant. The $\mathrm{H}_{0}$ represented that there was no difference of enamel print pattern on buccal and lingual surfaces for each tooth in an individual. Thus, the $\mathrm{H}_{0}$ was accepted, and the $H_{1}$ was rejected, which means that the enamel tooth prints on the buccal and lingual surfaces were pretty much the same.

\section{DISCUSSION}

Forensic odontology plays a significant role in the identification of remains, especially when there is traumatic tissue injury or lack of a fingerprint record which invalidate the use of visual or fingerprints methods. ${ }^{13}$ The principal advantage of dental evidence is that, like other hard tissues, it is often preserved after death. Teeth consist of enamel which is the hardest mineralized substance in the human body due to its superior organization and mineralization; hence, enamel prints are beneficial in personal identification in forensic odontology. ${ }^{14}$

Enamel does not remodel once it has been established and also does not remain in contact with the secretory cells or ameloblasts. Ameloblasts lay down enamel rods in an undulating and intertwining path and move or retract away from the enamel surface, leaving behind the prism morphology once enamel is formed. ${ }^{1}$ Thus, the study of enamel print patterns or also known as ameloglyphics is considered as a highly reliable biometric-based procedure for human identification. ${ }^{15}$

In this research, photomicrography technique is used to obtain the enamel print patterns. According to Ramenzoni ${ }^{16}$, the photomicrography technique was used in the study and enamel print pattern could be photographed without special preparation from extracted teeth. The enamel print patterns were visible from outside in the cervical to the mid coronal regions of the teeth. ${ }^{16}$ Hence, the use of photomicrograph technique is reliable and reduces the spoiling of enamel prints due to excess finger pressure in sample taking.

By using the photomicrograph technique, it is recorded that each of the samples in this research is made up of one or more than one sub-patterns. 
There are eight distinct sub-patterns recorded, namely wavy-branched, wavy-unbranched, linearbranched, linear-unbranched, whorl-open, whorlclosed, loop and stem-like. The sub-patterns can be defined, such as wavy-branched is the curve with branching while wavy-unbranched is a curve without branching. Linear-branched is a straight line with branching while linear-unbranched is a straight line without branching. Next, whorl-open and whorl-closed sub-patterns are similar to coiled shaped with an open border or closed border, respectively. Loop sub-pattern may also resemble loophole shape while stem-like sub-pattern seems like stem shape.

Researchers found that the enamel print patterns were distinct from each other either from different individuals or same individuals. ${ }^{16}$ The individualization of tooth print may be attributed to the unique variations in environmental factors surrounding each developing tooth which includes placement of the developing tooth bud, temperature, pressure and nutrition to the ameloblasts cells. Genetics might also have a role in predetermining the type of pattern. ${ }^{17}$

As we know, every tooth showed definite enamel rod end patterns which were specific for an individual and also specific for a tooth with dissimilarities between those of different individuals and also the same individual (comparison of incisor with incisor; canine with canine; premolar with premolar) ${ }^{2}$. However, this study was focusing on enamel tooth prints on different surfaces of the same tooth. Enamel print patterns of buccal and lingual were observed and compared for each of the samples respectively in order to obtain higher results accuracy.

The current research results showed that the same types of sub-patterns on the buccal and lingual surfaces on a tooth was high, indicating significant similarity between buccal and lingual surfaces. This result indicated that even though the enamel print patterns of buccal and lingual surfaces in the same sample may not have the same arrangement but the frequency of the similar sub-patterns occurs or absents on both surfaces were very high (Figure 4). This condition may be due to the same placement of developing tooth bud, temperature, pressure and nutrition to the ameloblasts cells in a tooth. The tooth receives similar environmental factors and genetics as well, leading it to have a similar possibility of sub-patterns in different surfaces in one tooth. Due to the high similarity between both buccal and lingual surfaces, lingual surfaces can be used as a choice when taking enamel print patterns as an antemortem record for dental identification in the forensic field. Enamel print patterns are unable to obtain if the teeth surfaces are tainted, for instances, wearing orthodontic appliances and composite restoration. Hence, lingual surfaces, which is the inner part of the tooth, are rarely used, thus makes a suitable substitution.

Furthermore, in an incident where enamel print patterns of buccal surface are no longer attainable, but there is only antemortem record of buccal surface, we can compare it with lingual surfaces as they have a high percentage of similarity. By doing so, we can narrow down the prospect of identifying an individual on a large scale. If the results show no significant similarity, the comparison of enamel print pattern in forensic identification could not be carried out to compare between two surfaces in the same tooth. However, the single surface of the tooth can still be used as identification with antemortem records.

According to the results presented in Figure 4, the most dominant sub-patterns found in this study were wavy-branched, followed by whorlopen, linear-branched. The less common subpattern was linear-unbranched and whorl-closed which have the same number of sub-pattern, followed by loop, wavy-unbranched and stem-like. In this research, 145 sub-patterns were found on 34 teeth of extracted permanent maxillary right first premolar. These results indicated that enamel prints on a surface were a combination of these sub-patterns. Each sample has a combination of a few sub-patterns, with a minimum of at least two sub-patterns. For example, more than one subpattern can be found on enamel print patterns on the buccal of a sample. The buccal surface of a tooth might be made up of wavy-branched, linear branched and whorl opened sub-patterns. This condition might become the reason why the number of sub-pattern was more than the number of samples. Other researchers also concluded that wavy-branched sub-pattern was the predominant sub-pattern. ${ }^{18}$ Enamel rod follows an undulating course from the dentinoenamel junction to the external tooth surface. Many rods span the entire 
thickness of enamel in a straight course, whereas most have a wavy pattern.

\section{CONCLUSION}

There is no difference between the enamel print patterns on the buccal and lingual surfaces of the extracted permanent maxillary right first premolar. Both buccal and lingual in the samples show high similarity due to the occurrence of similar sub-patterns on both surfaces are very high. The uniqueness of these enamel prints may be utilised as a successful personal identification tool in forensic science.

\section{REFERENCES}

1. Dinkar D, Siddarth P, Shravya M, Kishore B, Nayak SV, Raghavendra K, et al. Ameloglyphics - A mirror within you. Austin J Forensic Sci Criminol. 2018; 5(1): 1075.

2. Debnath N, Gupta R, Nongthombam RS, Chandran P. Forensic odontology. J Med Soc. 2016; 30(1): 20-3. DOI: 10.4103/09724958.175794

3. Deshmukh RA. Need for newer techniques for personal identification. J Forensic Res. 2015; 6(3): 284. DOI: 10.4172/2157-7145.1000284

4. Bansode SC, Kulkarni MM. Importance of palatal rugae in individual identification. 2009; 1(2): 77-81. DOI: 10.4103/0974-2948.60378

5. Sugunakar RGS, Rao TM, Nandan SRK, Kulkarni PG, Reddy SPD. Ameloglyphics - Can it aid in forensic identification. Indian J Dent Adv. 2014; 6(4): 1669-73. DOI: $10.5866 / 2014.641669$

6. Bharanidharan R, Karthik R, Rameshkumar A, Rajashree P, Rajkumar K. Ameloglyphics: An adjunctive aid in individual identification. SRM J Res Dent Sci. 2014; 5(4): 264-8. DOI: 10.4103/0976-433X.145147

7. Juneja $M$, Juneja $S$, Rakesh $N$, Kantharaj YDB. Ameloglyphics: A possible forensic tool for person identification following high temperature and acid exposure. J Forensic Dent Sci. 2016; 8(1): 28-31. DOI: 10.4103/09751475.176951

8. Sharma BS, Srivastav AK, Rajwar YC, Sharma SS, Sharma D, Batra P. Forensic: At first sight.
Ann Dent Spec. 2014; 2(2): 59-63.

9. Manjunath K, Saraswathi TR, Sriram G, Sundharam S, Porchelvam S. Reliability of automated biometrics in the analysis of enamel rod end patterns. J Forensic Dent Sci. 2009; 1(1): 32-6. DOI: 10.4103/09742948.50887

10. Manjunath K, Sivapathasundharam B. Analysis of enamel rod end pattern at different levels of enamel and its significance in ameloglyphics. J Forensic Res. 2014; 5(4): 235. DOI: $10.4172 / 2157-7145.1000235$

11. Howell DC. Statistical Methods for Psychology. 6th ed. California: Wadsworth Publishing; 2006. pp. 1-9.

12. Snape D, Spencer L. The Foundations of Qualitative Research. In: Ritchie J, Lewis J. Qualitative Research Practice. 1st ed. California: SAGE; 2003. pp. 349.

13. Avon SL. Forensic odontology: The roles and responsibilities of the dentist. 2004; 70(7): 453-8.

14. Shirish JP, Subrao BS. Study of enamel rod end patterns using acetate peel technique and automated biometrics for personal identification. Int J Dent Med Res. 2014; 1(4): 47-50.

15. White SN, Luo W, Paine ML, Fong H, Sarikaya $M$, Snead ML. Biological organization of hydroxyapatite crystallites into a fibrous continuum toughens and controls anisotropy in human enamel. J Dent Res. 2001; 80(1): 321-6. DOI: $10.1177 / 00220345010800010501$

16. Ramenzoni LL, Line SRP. Automated biometrics-based personal identification of the Hunter-Schreger bands of dental enamel. Proc Biol Sci. 2006; 273(1590): 1155-8. DOI: 10.1098/rspb.2005.3409

17. Gupta N, Jadhav K, Ahmed Mujib BR, Amberkar VS. Is re-creation of human identity possible using tooth prints? An experimental study to aid in identification. Forensic Sci Int. 2009; 192(1-3): 67-71. DOI: 10.1016/j. forsciint.2009.07.017

18. Dahal S, Boaz K, Srikant N, Agrawal NK. Ameloglyphics: A novel appraisal of the enamel structure in identification of an individual. $J$ Inst Med. 2014; 36(3): 64-7. 